\title{
CYLINDER WAKE STABILIZATION USING A MINIMAL ENERGY COMPENSATOR
}

\author{
Marco CARINI ${ }^{1}$, Jan Oscar PRALITS ${ }^{2}$ and Paolo LUCHINI ${ }^{3}$ \\ ${ }^{1}$ Dipartimento di Scienze e Tecnologie Aerospaziali, Politecnico di Milano \\ via La Masa 34, 20158 Milano, Italy. \\ ${ }^{2}$ Dipartimento di Ingegneria Chimica, Civile e Ambientale, Università degli Studi di Genova \\ via Montallegro 1, 16145 Genova, Italy. \\ ${ }^{3}$ Dipartimento di Ingegneria Industriale, Università degli Studi di Salerno \\ via Ponte don Melillo 1, 84084 Fisciano (SA), Italy.
}

\begin{abstract}
.
In the present work a linear feedback control strategy is used to control and suppress the cylinder vortexshedding at low Reynolds numbers. The classical minimal control energy or small gain solution of the optimal control and estimation problems is exploited in order to design a full-dimensional stabilizing compensator of the linearized Navier-Stokes equations. Both feedback and observer gains are efficiently computed based solely on the knowledge of the unstable adjoint and direct global modes, respectively. In our control setup, actuation is realized by means of angular oscillations of the cylinder surface while a single velocity sensor is employed for the state estimate. The derived compensator is shown to be able to drive the flow from the natural limit cycle to the unstable steady state which is finally restored. Then the sensitivity of the control performance to sensor placement and Reynolds number is investigated.
\end{abstract}

Key words: bluff-bodies, incompressible flow, global instabilities, linear optimal control.

\section{Introduction}

The control of the vortex shedding occurring in the wake of a bluff-body represents a great challenge in many engineering applications. The inherent low frequency unsteadiness of the flow field is indeed source of noise, structural vibrations and resonance, and can be reduced or even suppressed by a suitable control action. A lot of works have been dedicated to this subject and several strategies have been proposed as documented by the review of Choi et al. [6]. In particular, besides passive devices and open-loop techniques, active feedback controls have attracted an increasing attention due to their ability to adapt to the actual flow conditions.

During the past decade a model-based approach to flow control has been established within the framework of linear dynamical systems and optimal control theory [11] with the fluid plant being derived from the linearized description of growing/decaying instabilities around the given base flow [2,1]. Within this framework, the classical minimal control energy (MCE) (or small gain) solution of the optimal control problem has been recently reviewed by Lauga and Bewley [12] and Bewley et al. [4] in view of its application to large scale models of globally unstable flows. In particular the latter authors 
have proposed an efficient technique to compute the related MCE feedback rule based solely on the knowledge of the unstable adjoint eigenspace whose dimension is found to be small for typical fluid applications.

In the present work this latter approach is adopted in order to design a full-dimensional stabilizing compensator of the flow past a circular cylinder. Starting from the work of Bewley et al. [4], the MCE solution of the linear optimal estimation problem is also considered, leading to the design of a Linear Quadratic Gaussian (LQG) compensator in the limiting case of minimal control effort and extremely noisy measures (Burl, 1999). For such a compensator any exerted control and estimation effort concentrates on stabilizing and estimating only the unstable modes of the uncontrolled system. Then the MCE control strategy is applied to the suppression of the first instability of the cylinder wake [9]. This flow is indeed prototypical of bluff-body wakes as well as of fluid oscillators from global stability viewpoint. Several control studies aimed to suppress or mitigate the cylinder vortex shedding have been described in the past literature $[19,16,10,18,3]$ but only few works have addressed this problem within the linear optimal control framework: an example is provided by the work of Protas [17]. In the present study control actuation is realized by means of angular oscillations of the cylinder surface while a single velocity sensor is used for the state estimate. A similar control setup has been employed by Fujisawa and Nakabayashi [8] in their experiments at higher Reynolds numbers achieving a maximum $16 \%$ of drag reduction at $\mathrm{Re}=20000$. Nevertheless the proposed MCE strategy is not limited to the particular control configuration adopted herein. Direct numerical simulations (DNS) of the controlled nonlinear flow are performed to assess the effectiveness of the MCE strategy. Variations of the control performance with respect to sensor placement as well as its dependence on Reynolds number are investigated.

\section{Problem definition}

The two-dimensional flow around a circular cylinder is described herein by means of a Cartesian coordinate system whose origin is coincident with the cylinder centre and whose $x$-axis is aligned to the flow direction. The fluid motion is governed by the incompressible Navier-Stokes equations which are made dimensionless using the cylinder diameter $D^{*}$, the velocity of the incoming stream $V_{\infty}^{*}$ and the constant density $\rho^{*}$ :

$$
\left\{\begin{array}{l}
\frac{\partial \boldsymbol{V}}{\partial t}+(\boldsymbol{V} \cdot \boldsymbol{\nabla}) \boldsymbol{V}=-\boldsymbol{\nabla} P+\frac{1}{\mathrm{Re}} \nabla^{2} \boldsymbol{V}, \\
\boldsymbol{\nabla} \cdot \boldsymbol{V}=0
\end{array}\right.
$$

where $\boldsymbol{V}$ denotes the velocity vector with components $\boldsymbol{V}=(U, V), P$ is the reduced pressure and $\operatorname{Re}=V_{\infty}^{*} D^{*} / \nu$ is the Reynolds number ( $\nu$ being the kinematic viscosity of the fluid). The above equations are solved on the rectangular domain $\Omega_{c}$ which is illustrated in Figure 1 where the control setup is also sketched. On $\Omega_{c}$ the equations (1) are supplemented by a zero normal stress condition at the outlet boundary $\Gamma_{\text {out }}$ while at the inlet $\Gamma_{\text {in }}$ the vorticity is set to zero and a uniform streamwise velocity profile is assigned $(U=1)$. Similarly, on the upper and lower boundaries $\Gamma_{\text {top }}$ and $\Gamma_{\text {bottom }}$, respectively, both the normal velocity component and the vorticity are assumed to vanish. As already mentioned, for the considered flow configuration the control variable is represented by the angular velocity $\varphi(t)$ of the cylinder rotating around its axis in the counter-clockwise 


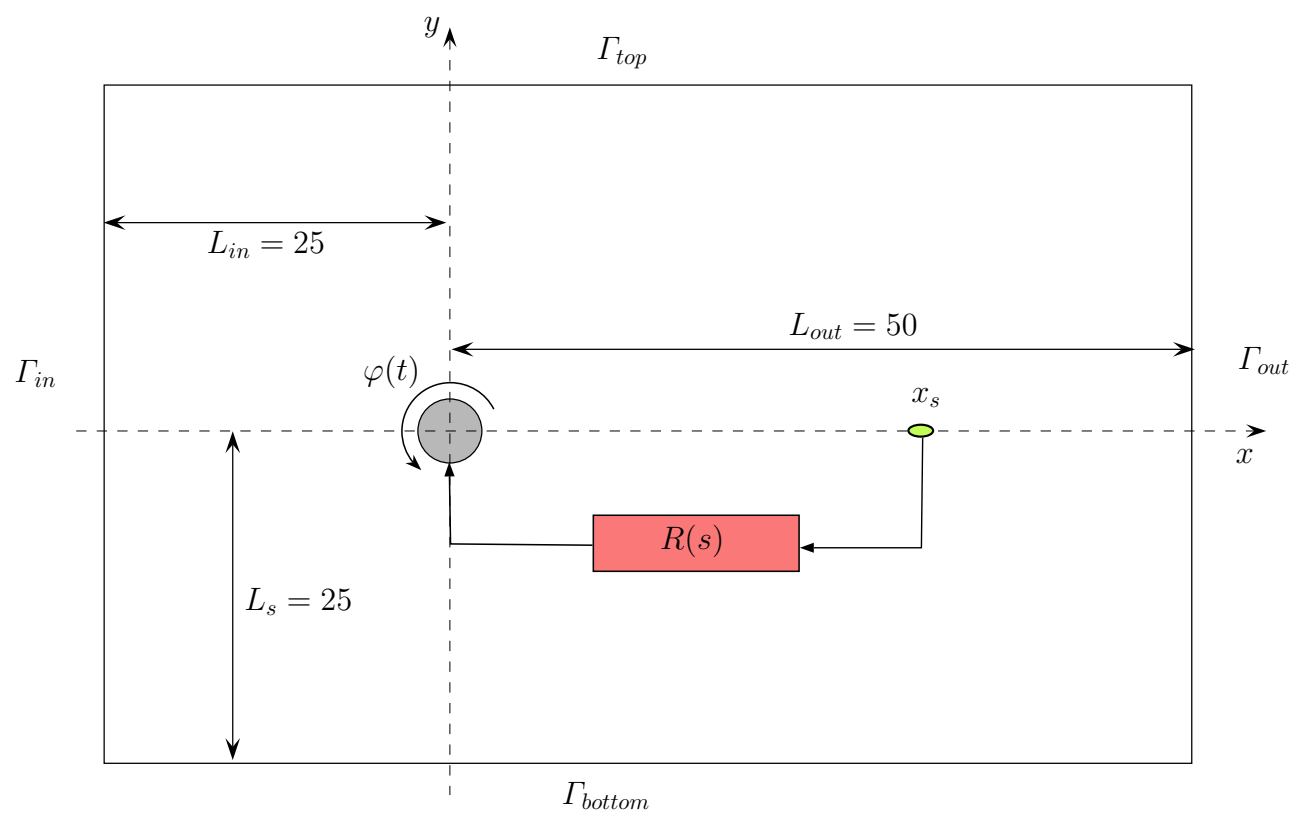

Figure 1: Sketch of the computational domain $\Omega_{c}$ employed for the numerical simulation of the flow past the circular cylinder with the adopted control setup. The local velocity sensor is represented by the green "dot" located at the streamwise station $x_{s}$ along the $x$-axis while $R(s)$ is used to indicate the related SISO compensator.

direction. This corresponds to impose $\boldsymbol{V}=1 / 2 \varphi \boldsymbol{\tau}$ on the cylinder surface $\Gamma_{c}, \boldsymbol{\tau}$ being the unit tangent vector.

The definition of the Linear Time Invariant (LTI) fluid plant for control design is easily derived by linearizing the Navier-Stokes equations around the base flow $\boldsymbol{Q}_{b}=\left\{\boldsymbol{V}_{b}, P_{b}\right\}$ which is solution of the steady version of (1) for $\varphi=0$. The governing equations for the evolution of the perturbation field $\boldsymbol{q}=\{\boldsymbol{v}, p\}$ are given by:

$$
\left\{\begin{array}{l}
\frac{\partial \boldsymbol{v}}{\partial t}+\mathcal{L}\left\{\boldsymbol{V}_{b}, \operatorname{Re}\right\} \boldsymbol{v}+\nabla p=\mathbf{0}, \\
\boldsymbol{\nabla} \cdot \boldsymbol{v}=0
\end{array}\right.
$$

where $\mathcal{L}\left\{\boldsymbol{V}_{b}, \operatorname{Re}\right\}$ stands for the linearized Navier-Stokes operator:

$$
\mathcal{L}\left\{\boldsymbol{V}_{b}, \operatorname{Re}\right\} \boldsymbol{v}=\left(\boldsymbol{V}_{b} \cdot \boldsymbol{\nabla}\right) \boldsymbol{v}+(\boldsymbol{v} \cdot \boldsymbol{\nabla}) \boldsymbol{V}_{b}-\frac{1}{\operatorname{Re}} \nabla^{2} \boldsymbol{v}
$$

The same boundary conditions imposed on $\partial \Omega_{c}$ for $\boldsymbol{V}$ will hold for $\boldsymbol{v}$ in homogeneous form except on $\Gamma_{c}$ where the control actuation is realized. Once spatially discretized, equations (2) can be recast in the so-called descriptor form:

$$
E \frac{d \boldsymbol{x}}{d t}=A \boldsymbol{x}+B u,
$$

where $\boldsymbol{x}$ and $A$ correspond to the discrete counterpart of $\boldsymbol{q}$ and $\mathcal{L}\left\{\boldsymbol{V}_{b}, \operatorname{Re}\right\}$, respectively, while $E$ denotes the singular mass matrix and $u=\varphi(t)$. When control is turned off, i.e. for $\varphi=0$, equations (2) describe the natural evolution of linear flow perturbations which corresponds to the open-loop dynamics from the control viewpoint. The inherent 
global spectrum and the related global modes are computed via the common assumption of exponential time-dependence:

$$
\boldsymbol{q}(x, y, t)=\hat{\boldsymbol{q}}(x, y) \exp (\lambda t) .
$$

The above ansatz leads to a generalized eigenvalue problem for $\lambda$ and for the non trivial complex valued field $\hat{\boldsymbol{q}}(x, y)$ that, in discrete form, reads

$$
A \hat{\boldsymbol{x}}=\lambda E \hat{\boldsymbol{x}} .
$$

For the flow past a circular cylinder, it is well known that a pair of complex-conjugate eigenvalues becomes unstable above the critical threshold of $\operatorname{Re} \sim 47$ and the related direct and adjoint global modes have been described by several authors $[9,15]$. The same results will be used herein in order to design the stabilizing MCE compensator of the cylinder wake.

\subsection{The MCE compensator}

It is a classical result in linear optimal control theory that when the MCE limit is taken, the feedback rule $u=K \boldsymbol{x}$ leads to the reflection of the unstable eigenvalues of $A$ across the imaginary axis, while leaving unchanged all the remaining stable modes. For such a solution, the feedback gain matrix $K$ can be exactly computed by exploiting the particular structure of the closed-loop spectrum, i.e. the spectrum of $A+B K$, which is known $a$ priori. An analytical formula for $K$ bypassing the solution of the Riccati equation has been derived by Lauga and Bewley [12]:

$$
K=-R^{-1} B_{u}^{H} F^{-1} P_{u}^{H} E,
$$

where $P_{u}$ denotes the unstable left eigenvectors of $A$, i.e. $P_{u}^{H} A=\Lambda_{u} P_{u}^{H} E, B_{u}=P_{u}^{H} B$ and $R>0$ corresponds to the control weight matrix within the cost function definition:

$$
J=\frac{1}{2} \int_{0}^{\infty}\left(\boldsymbol{x}^{H} Q \boldsymbol{x}+\boldsymbol{u}^{H} R \boldsymbol{u}\right) d t
$$

The matrix $F$ in equation (7) is simply defined as

$$
F_{i j}=M_{i j} /\left(\lambda_{i}+\lambda_{j}^{*}\right), \quad \text { with } \quad M=B_{u} R^{-1} B_{u}^{H} .
$$

Thus the solely knowledge of the unstable eigenvalues $\Lambda_{u}$ and of the left (or adjoint) eigenvectors $P_{u}$ is required for the computation of $K$. Similarly to the control case, the above MCE approach can be employed for the observer design within the common framework of Kalman filtering [14, 5]. For the linear optimal estimation problem, plant equations are re-written in the form:

$$
\begin{aligned}
E \frac{d \boldsymbol{x}}{d t} & =A \boldsymbol{x}+B \boldsymbol{u}+\boldsymbol{d} \\
\boldsymbol{y} & =C \boldsymbol{x}+\boldsymbol{r}
\end{aligned}
$$

where the output relation for the available plant measures $\boldsymbol{y}$ has been introduced along with the measurement noise $r$ and the state disturbance $\boldsymbol{d}$. According to the Kalman filtering theory, a stochastic description of both $\boldsymbol{d}$ and $\boldsymbol{r}$ is assumed, with $\boldsymbol{d}$ and $\boldsymbol{r}$ being modeled as uncorrelated, zero mean, white Gaussian processes with known covariance 
matrices $W_{d d}=\mathcal{E}\left\{\boldsymbol{d} \boldsymbol{d}^{H}\right\}$ and $W_{r r}=\mathcal{E}\left\{\boldsymbol{r} \boldsymbol{r}^{H}\right\}$, respectively ${ }^{1}$. The linear observer which governs the approximation $\boldsymbol{o}$ to the true state $\boldsymbol{x}$ is defined as

$$
\begin{aligned}
E \frac{d \boldsymbol{o}}{d t} & =A \boldsymbol{o}+B \boldsymbol{u}-L\left(\boldsymbol{y}-\boldsymbol{y}_{o}\right) \\
\boldsymbol{y}_{o} & =C \boldsymbol{o}
\end{aligned}
$$

where the matrix $L$ of observer gains is referred to as the Kalman gain matrix and it is optimally designed in order to minimize the expected 'energy' $\mathcal{E}\left\{\boldsymbol{e}^{H} \boldsymbol{e}\right\}$ of the estimation error $\boldsymbol{e}=\boldsymbol{x}-\boldsymbol{o}$. At this point it is worthwhile to note that $L$ can be equivalently computed as the solution of the optimal control problem on the so called dual system of (4)

$$
E \frac{d \tilde{\boldsymbol{p}}}{d t}=A^{H} \tilde{\boldsymbol{p}}+C^{H} \tilde{\boldsymbol{r}}
$$

with the cost function

$$
J_{e}=\frac{1}{2} \int_{0}^{\infty}\left(\tilde{\boldsymbol{p}}^{H} W_{d d} \tilde{\boldsymbol{p}}+\tilde{\boldsymbol{r}}^{H} W_{r r} \tilde{\boldsymbol{r}}\right) d t
$$

When the MCE solution of the dual control problem is considered, this corresponds to the classical Kalman filter design in the limiting case of extremely noisy measures compared to modeling errors on the state dynamics. In this case the matrix $L$ can be easily obtained by applying the MCE formula (7) to the dual control problem:

$$
L=-E X_{u} G^{-H} C_{u}^{H} W_{r r}^{-1},
$$

where $X_{u}$ denotes the unstable right (or direct) eigenvectors of $A$, i.e. $A X_{u}=X_{u} \Lambda_{u}$, $C_{u}=C X_{u}$ and the matrix $G$ is defined as

$$
G_{i j}=N_{i j} /\left(\lambda_{i}^{*}+\lambda_{j}\right), \quad \text { with } \quad N=C_{u}^{H} W_{r r}^{-1} C_{u} .
$$

Once the MCE solution of both the control and the estimation problems is considered, the MCE formulation of the LQG compensator is obtained [5]. For such a particular LQG compensator any control and estimation effort will be focused on stabilizing and estimating only the unstable modes of the uncontrolled system. By exploiting the above results, the related gain matrices $K$ and $L$ are computed based solely on the knowledge of the unstable eigenspace of $A$. For typical fluid applications aimed to suppress global instabilities, the number of unstable modes is very small compared to the state dimension, thus making the design of the MCE-LQG compensator computationally feasible and efficient even for those large scale systems originating from the numerical discretization of the Navier-Stokes equations.

\subsection{Numerical methods}

The two-dimensional Navier-Stokes equations (1) and their linearized version (2) are discretized in conservative form on Cartesian smoothly varying staggered grids using standard second-order finite difference schemes. An immersed boundary technique is employed to impose the considered boundary conditions on $\Gamma_{c}$ [see 9 , for details] and a bilinear interpolation is used to sample the velocity field at the selected sensor position.

\footnotetext{
${ }^{1} \mathcal{E}\{\cdot\}$ is employed herein to denote the expectation operator
} 


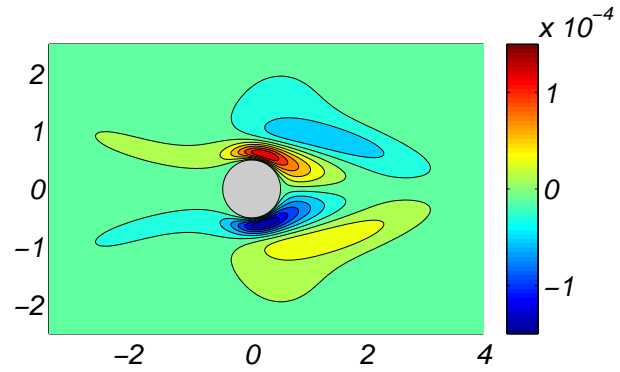

(a)

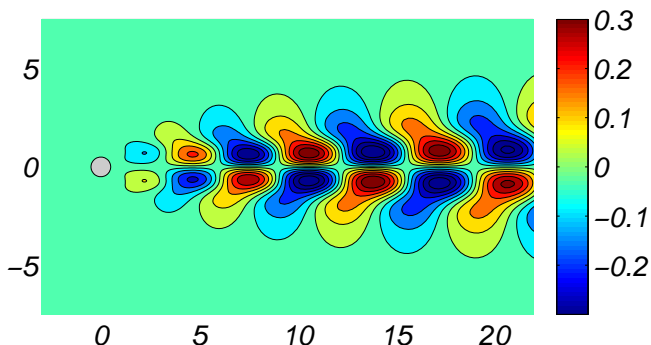

(b)

Figure 2: MCE gain fields at $R e=50$ : continuous representation as two-dimensional vector fields. (a) Streamwise component of the controller gain field $\mathcal{K}_{u}(x, y)$. (b) Streamwise component of the observer gain field $\mathcal{L}_{u}(x, y)$ with reference to a single cross-stream velocity sensor located at $(x, y)=(1.0,0.0)$.

Thus the non-zero entries of $B$ and $C$ are defined accordingly to the above interpolation procedures. Classical Newton iterations are used to compute the steady base flow $\boldsymbol{Q}_{b}$ while the generalized eigenvalue problem (6) is solved by means of the Implicitly Restarted Arnoldi method, implemented in the ARPACK library [13]. Time integration of the semi-discretized linear and nonlinear equations is performed making use of the Adams-Bashforth/Crank-Nicolson scheme: the diffusive terms and the pressure field are treated implicitly and at each time step a Stokes-like operator is numerically inverted using the sparse LU solver provided with the free software package UMFPACK [7]. The same linear solver is employed to handle all other required matrix inversions in our computational setup. Both the term $B K \boldsymbol{x}$ for the controlled system and the term $L \boldsymbol{y}$ for the compensator dynamics are integrated explicitly thus avoiding the storage and factorization of the huge matrix associated with the closed-loop dynamics.

All the presented results have been computed on a domain $\Omega_{c}$ of length $L_{x}=75$ in the streamwise direction and $L_{y}=50$ in the cross-stream direction. With reference to Figure 1 , the inlet, the outlet and the lateral boundaries are located at a distance from the origin equal to $L_{\text {in }}=25, L_{\text {out }}=50$ and $L_{s}=25$ respectively. The whole computational domain is discretized using $450 \times 300$ nodes with grid points clustered near the cylinder surface. More precisely, a uniform mesh with the finest grid spacing of $\Delta x=\Delta y=0.02$ is adopted within the small rectangular subdomain $[-1,1] \times[-1,1]$ enclosing the cylinder. Finally for time integration, a non-dimensional step of $\Delta t=0.01$ is employed.

\section{Results}

The MCE control strategy is now applied to the cylinder flow at $\operatorname{Re}=50$. As already mentioned, when the Reynolds number is increased beyond the critical value of $\operatorname{Re} \sim 47$, the steady base flow becomes linearly unstable with a pair of complex-conjugate modes. In particular at $\operatorname{Re}=50$ we found $\lambda_{u}=0.0139 \pm 0.736 i$. The computed MCE gain matrix $K$ is illustrated in Figure 2a as a continuous two-dimensional vector field by means of its streamwise component $\mathcal{K}_{u}(x, y)$. In agreement with the prior results of Bewley et al. [4], the gain spatial distribution is sharply localized close to the cylinder surface and appears very similar to that of the unstable adjoint global mode of the cylinder wake $[9,15]$. This 


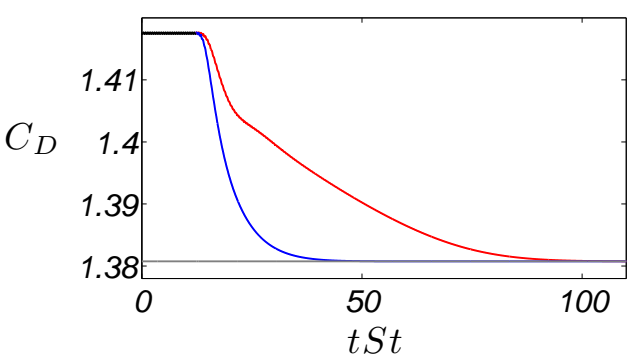

(a)

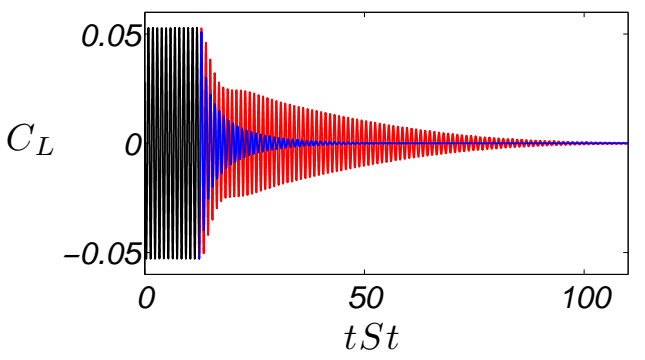

(c)

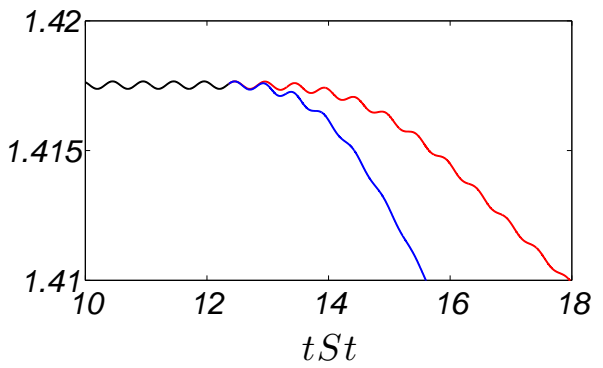

(b)

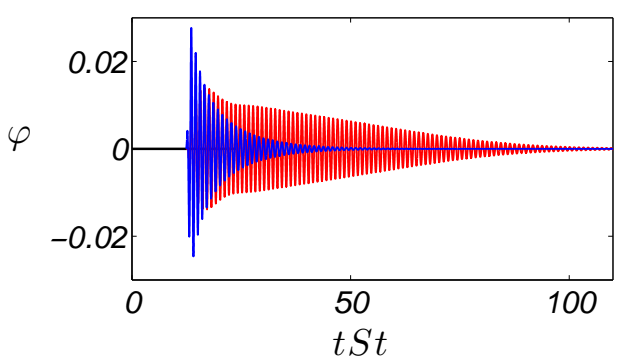

(d)

Figure 3: MCE control of the flow past a circular cylinder at $\mathrm{Re}=50$ : time traces of the drag coefficient $C_{D}$ (a)-(b), of the lift coefficient $C_{L}$ (c) and of the cylinder angular velocity $\varphi(t)$ (d). In the above plots, the nondimensional time $t S t$ is employed with $S t$ being the Strouhal number associated with the uncontrolled shedding cycle. Control starts on the fully developed limit cycle (black line). The performance of the MCE compensator using a single cross-wise velocity sensor located at $\left(x_{s}, y_{s}\right)=(1.0,0)$ (red line) is compared with that of the full-information controller (blue line). The gray line in Figure (a) denotes the value of $C_{D}$ associated with the steady base flow

can be better understood by observing from equation (7) that $K$ can be re-written as a linear combination of the real and imaginary part of the unstable left eigenvector $\hat{\boldsymbol{p}}_{u}$ :

$$
K=\gamma_{r} \Re\left(\hat{\boldsymbol{p}}_{u}\right)+\gamma_{i} \Im\left(\hat{\boldsymbol{p}}_{u}\right),
$$

where $\gamma_{r}$ and $\gamma_{i}$ are two real valued coefficients. Similar considerations hold also for the Kalman gain matrix that has been computed with reference to a single cross-stream velocity sensor located at $\left(x_{s}, y_{s}\right)=(1,0)$. The field continuous representation of $L$ by means of its streamwise component $\mathcal{L}_{u}(x, y)$ is illustrated in Figure 2b. Not surprisingly the spatial distribution of the observer gain field is found very similar to that of the direct unstable global mode. Indeed from equation (14), $L$ results from a linear combination of the real and imaginary part of the unstable right eigenvector $\hat{\boldsymbol{x}}_{u}$ in a dual manner to equation (16). In addition with reference to the expressions (7) and (14), it can be easily shown that in the (present) single input single output (SISO) case the numerical values of $R$ and $W_{r r}$ do not affect the resulting feedback and observer gains; therefore in the following we will simply assume $R=W_{r r}=1$. The obtained solutions for $K$ and $L$ have been validated on the linear plant by evaluating both the growth-rate and frequency of the least stable modes of the closed-loop systems $A+B K$ and $A+L C$ which should correspond to the reflected unstable eigenvalues of $A$.

Then the effectiveness of the proposed MCE compensator is assessed on the fully nonlinear cylinder flow. Starting from the fully developed shedding cycle, both the fullinformation feedback controller and the derived SISO compensator are shown to be able 


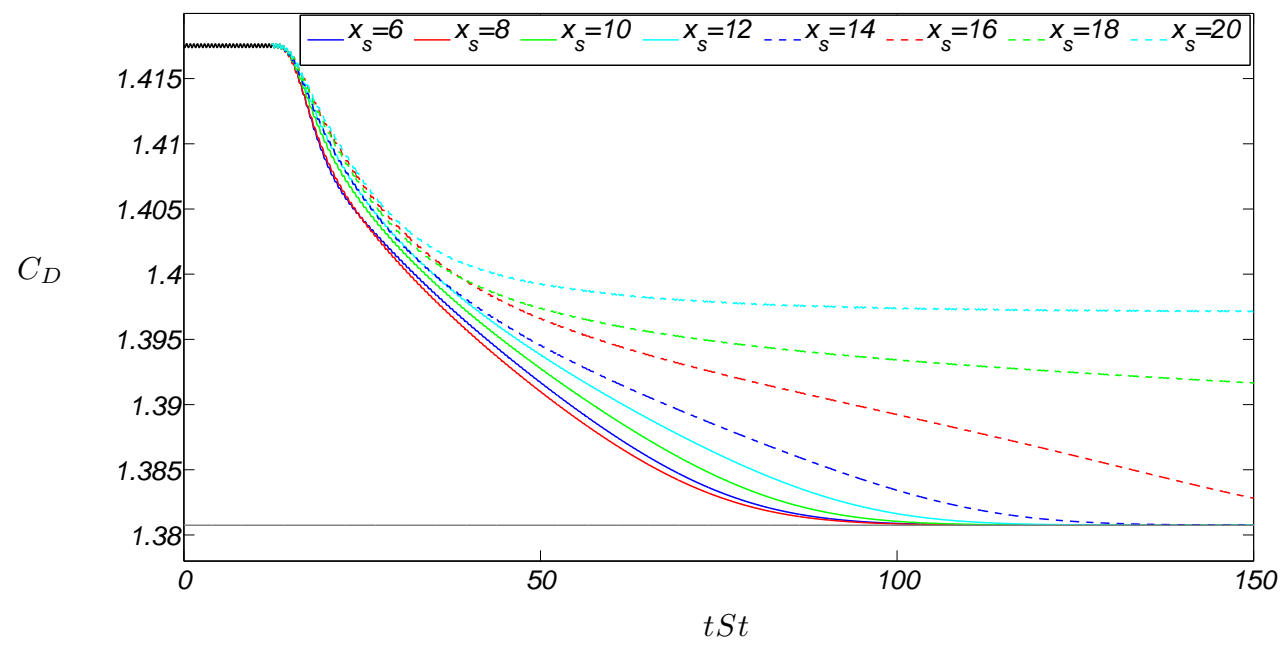

Figure 4: MCE control of the flow past a circular cylinder at $\mathrm{Re}=50$ using a single measure of the crossstream velocity component along the $x$-axis. Time traces of the drag coefficient $C_{D}$ for different streamwise location $x_{s}$ of the sensor.

to drive the flow towards the unstable state $\boldsymbol{Q}_{b}$ which is finally restored. This is clearly illustrated in Figure 3 by means of the time traces of the aerodynamic force coefficients and the control variable $u=\varphi(t)$. In Figure 3 as well as in the following representations, the nondimensional time $t$ is rescaled using the Strouhal number $S t$ associated with the uncontrolled vortex shedding, thus providing a measure of the equivalent number of vortex shedding cycles required to stabilize the flow. For both the full-information controller (blue line) and the compensator (red line), control is turned on at the same time instant and smoothly applied to the flow system by means of a blended step function. As expected, results in Figure 3 indicate that the full-information controller performs better than the compensator by achieving flow stabilization over a shorter time window ( $\sim 40$ cycles) compared to the second ( $\sim 100$ cycles). Indeed it is quite obvious that for the compensator a certain amount of time is spent for state estimation and only once the 'linear' flow state has been adequately reconstructed, the control becomes effective.

\subsection{Sensitivity to sensor placement \& Reynolds number}

In the present control setup only a single measure of the cross-stream velocity component along the $x$-axis has been used for state estimate. This sensor configuration is mainly suggested by the symmetry-breaking nature of the flow instability with respect to the steady state. The same sensor configuration has been adopted by [16] achieving a complete suppression of the cylinder vortex shedding up to $R e=60$. Furthermore in his experiments Roussopoulos [19] has shown that away from the centerline, the unsteadiness caused by vortex shedding is too weak to be used as a control signal.

Different streamwise location $x_{s}$ of the sensor have been tested and some of these results are illustrated in Figure 4 with reference to the $C_{D}$ time trace. When the sensor is moved downstream, the stabilization process becomes slower and, consequently, the amount of time required for the complete suppression of the vortex shedding increases. Moreover for a sensor placement in the far-wake region, i.e. $x_{s} \geq 19$, vortex shedding suppression cannot be achieved any more and the controlled flow converges to a different limit cycle 


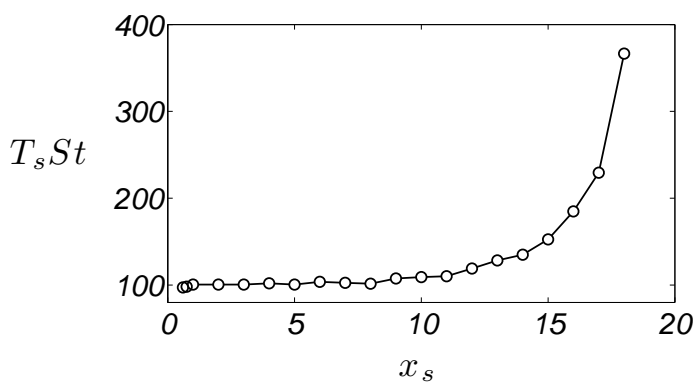

(a)

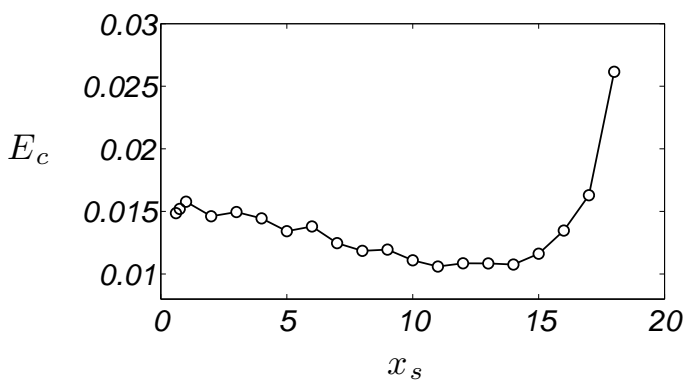

(b)

Figure 5: MCE control of the flow past a circular cylinder at $\mathrm{Re}=50$ using a single measure of the crossstream velocity component along the $x$-axis. (a) Stabilization time $T_{s}$ as a function of $x_{s}$. (b) Total control energy $E_{c}=\int_{0}^{T_{s}} \varphi^{2} d t$ as a function of $x_{s}$.

which is however characterized by a lower mean value of the drag coefficient. For practical purpose the time required to suppress the cylinder vortex shedding $T_{s}$ can be evaluated based on the amplitude of the drag coefficient fluctuations with respect to the base flow value, i.e. $\left\|C_{D}(t)-C_{D}^{(B F)}\right\| \leq \epsilon$. In Figure 5a the value of $T_{s}$ (with $\epsilon=10^{-5}$ ) is plotted as a function of $x_{s}$ : the time spent to stabilize the flow is shown to be weakly dependent on $x_{s}$ up to $x_{s} \sim 10$ after which $T_{s}$ rapidly increases. In Figure $5 \mathrm{~b}$ the total control energy $E_{c}=\int_{0}^{T_{s}} \varphi^{2} d t$ spent to stabilize the flow is also plotted as a function of $x_{s}$. These latter results indicate that the value of $E_{c}$ is approximately reduced to a minimum when the sensor is located at $x_{s} \sim 11-14$.

In view of more realistic control setups, the velocity sensor can be moved close to the cylinder surface. For such purpose we also consider sensor placement at a fixed radial distance of $\Delta r=0.1$ from the cylinder surface while varying the angular position $\theta$. For each sensor configuration at a different $\theta$ station, the radial velocity component is measured and the related MCE control performance is shown in Figure 6. The comparison with the results obtained for $\theta=0$, i.e. along the $x$-axis measuring the $v$ component of the velocity, indicates that no substantial improvement is obtained when the sensor is located at $\theta$ different from zero.

Finally the effectiveness of the MCE control at increasing Reynolds numbers is investigated up to $\operatorname{Re}=80$. At each considered value of $\mathrm{Re}$ and for both the full-information feedback controller and the SISO compensator, the achievement or not of the vortex shedding suppression is reported in Table 1. It is interesting to note that while the fullinformation controller is able to stabilize the flow up to $\mathrm{Re}=70$, the compensator already fails at $\operatorname{Re}=60$ even when the sensor is located very close to cylinder surface. i.e. at $x_{s}=0.6$. In particular at $\mathrm{Re}=70$ the control action of the MCE compensator drives the flow to a limit cycle characterized by higher mean value and fluctuation amplitude of the $C_{D}$. This is illustrated in Figure $7 \mathrm{~b}$ while in Figure $7 \mathrm{a}$ for $\mathrm{Re}=65$ the MCE compensator action still results in a small beneficial effect over the uncontrolled flow.

\section{Conclusions}

In this paper the feedback control of the cylinder wake is numerically investigated at low Reynolds numbers. Actuation is realized by means of cylinder angular oscillations while 


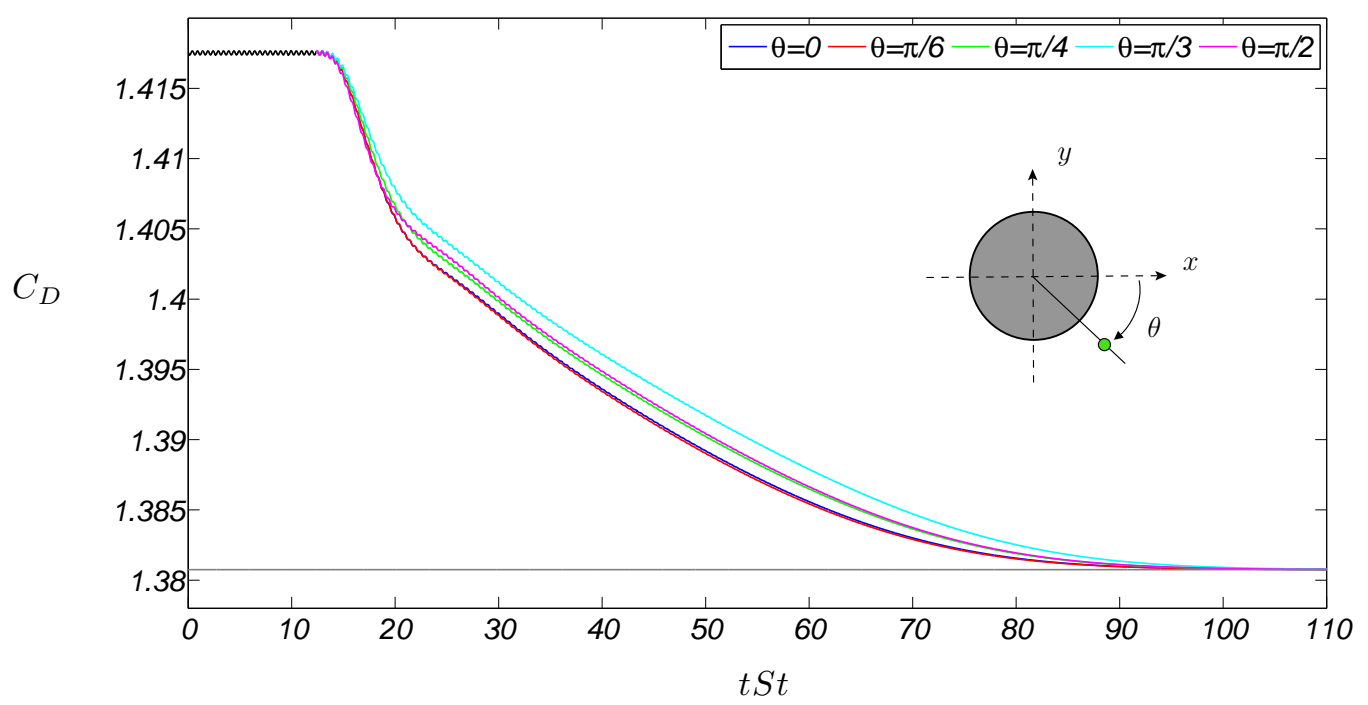

Figure 6: MCE control of the flow past a circular cylinder at $\mathrm{Re}=50$ using a single sensor of the radial velocity component located at a fixed distance $\Delta r=0.1$ from the cylinder surface. Time traces of the drag coefficient $C_{D}$ for different angular position $\theta$ of the sensor. At $\theta=0$ the $v$ component of the velocity is employed.

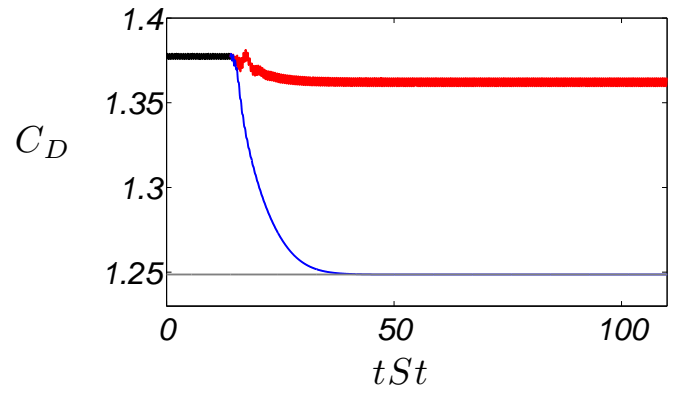

(a)

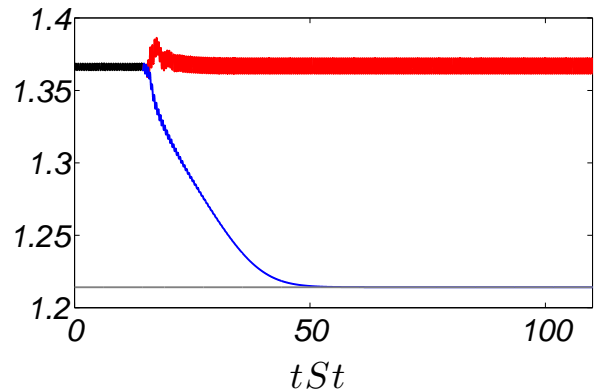

(b)

Figure 7: MCE control of the flow past a circular cylinder using a single cross-stream velocity sensor located at $x_{s}=0.6$. (a) $\mathrm{Re}=65$. (b) $\mathrm{Re}=70$. The performance of the MCE compensator (red line) is compared to that of the full-information controller (blue line) in terms of the time trace of the drag coefficient. Control starts on the fully developed limit cycle (black line). The gray line denotes the value of $C_{D}$ associated with the steady base flow. 


\begin{tabular}{cclll}
$\operatorname{Re}$ & $\lambda_{u}$ & Full-information & Compensator & $x_{s}$ \\
\hline 55 & $0.0318 \pm 0.741 i$ & Stabilized & Stabilized & 1.0 \\
60 & $0.0476 \pm 0.744 i$ & Stabilized & Not stabilized & 0.6 \\
65 & $0.0614 \pm 0.745 i$ & Stabilized & Not stabilized & 0.6 \\
70 & $0.0737 \pm 0.745 i$ & Stabilized & Not stabilized & 0.6 \\
75 & $0.0846 \pm 0.743 i$ & Not stabilized & Not stabilized & 0.6 \\
80 & $0.0943 \pm 0.741 i$ & Not stabilized & Not stabilized & 0.6 \\
\hline
\end{tabular}

Table 1: MCE control of the cylinder flow at increasing Reynolds numbers: comparison between the full-information controller and the SISO compensator performance with respect to the achievement of flow stabilization i.e. the complete vortex shedding suppression.

a single velocity sensor is employed for the state estimate. A full-dimensional MCELQG compensator of the linearized flow equations is designed and tested. Both feedback and observer gains are efficiently computed by exploiting the analytical result of Lauga and Bewley [12], thus requiring the solely knowledge of the unstable direct and adjoint eigenmodes. At $\mathrm{Re}=50$ when the single cross-stream velocity sensor is located along the $x$-axis in the near-wake, the cylinder vortex shedding is completely suppressed and the unstable base flow is finally restored. When the sensor is moved downstream the time required to stabilize the flow starts to gradually increase up to $x_{s}>10$ when it rapidly grows up until the control effectiveness is definitely lost for $x_{s} \geq 19$ (Figures 4-5). Besides the nonlinear evolution of the vortex street, this behavior can be physically interpreted based on the phase lag associated with the convective nature of the flow, which becomes more relevant as the sensor is moved far away from the cylinder surface where the control is actuated. The stabilization loss associated with a far-wake sensor placement was described also by Roussopoulos [19] in his control experiments at $\operatorname{Re}=65$ : in that case a critical threshold of approximately 9 diameters from the cylinder was found. For the considered streamwise sensor placement the amount of control energy spent to stabilize the flow is characterized by a small lower plateau when the sensor is located at $x_{s} \sim 11-14$. This sensor location roughly corresponds to the region where the maxima of the unstable direct global mode are found at $\mathrm{Re}=50$ [9]. It is worthwhile to note that the optimal placement for the cheapest control, i.e. for a minimum value of $E_{c}$, does not correspond also to the fastest stabilizing control for which the sensor should be moved close to cylinder surface (Figure 5a). The MCE control performance with a sensor placement very close to the cylinder surface and at various angular positions has been investigated. For such configurations the radial velocity component is measured. However obtained results at the different angular positions do not show any improvement with respect to those obtained using a $v$-component sensor placed along the $x$-axis and at the same distance from the cylinder surface; moreover control performance is slightly reduced. Finally the dependence of the MCE control performance from the Reynolds number have been addressed. At $\mathrm{Re}=60$ the MCE compensator is not able to stabilize the flow and at $\mathrm{Re}=70$ its control action results in a worsening of the flow instability with respect to the uncontrolled case, leading to an increase of the mean drag and of the aerodynamic load fluctuations. At the same time the full-information control is shown to 
be able to completely suppress the vortex shedding up to $\operatorname{Re}=70$, thus suggesting that the limited compensator performance is due to a poor linear estimate of the flow state.

\section{References}

[1] S. Ahuja and C. W. Rowley. Feedback control of unstable steady state of flow past a flat plate using reduced-order estimators. J. Fluid Mech., 645:447-478, 2010.

[2] A. Barbagallo, D. Sipp, and P.-J. Schmid. Closed-loop control of an open cavity flow using reducedorder models. J. Fluid Mech., 641:1-50, 2009.

[3] M. Bergmann, L. Cordier, and J. P. Brancher. Optimal rotary control of the cylinder wake using proper orthogonal decomposition reduced-order model. Phys. Fluids, 17(097101):1-21, 2005.

[4] T. R. Bewley, J. O. Pralits, and P. Luchini. Minimal-energy control feedback for stabilization of bluffbody wakes. Proceeding FEDSM2002-31048. In BBVIV5, Fifth Conference on Bluff Body Wakes and Vortex-Induced Vibrations, Bahia, Brazil, December 2007.

[5] J. B. Burl. Linear Optimal Control: $\mathcal{H}_{2}$ and $\mathcal{H}_{\infty}$ methods. Addison-Wesley, 1999.

[6] H. Choi, W. Jeon, and J. Kim. Control of flow over bluff body. Annu. Rev. Fluid Mech., 40:113-139, 2008.

[7] T. A. Davis. Algorithm 832: UMFPACK, an unsymmetric-pattern multifrontal method. ACM Trans. Math. Software, 30(2):196-199, 2004.

[8] N. Fujisawa and T. Nakabayashi. Neural network control of vortex shedding from a circular cylinder using rotational feedback oscillations. J. Fluids and Struct., 16:113-119, 2002.

[9] F. Giannetti and P. Luchini. Structural sensitivity of the first instability of the cylinder wake. J. Fluid. Mech., 581:167-197, 2007.

[10] J.-W. He, R. Glowinski, R. Metcalfe, A. Nordlandelr, and J. Periaux. Active control and drag optimization for flow past a circular cylinder: I. Oscillatory cylinder rotation. J. Comp. Phys, 163:83-117, 2000.

[11] J. Kim and T. R. Bewely. A linear system approach to flow control. Annu. Rev. Fluid Mech., 39: 383-417, 2007.

[12] E. Lauga and T. R. Bewley. The decay of stabilizability with Reynolds number in a linear model of spatially developing flows. Proc. R. Soc. Lond. A, 459:2077-2095, 2003.

[13] R. B Lehoucq, D. C. Sorensen, and C. Yang. ARPACK Users Guide, siam edition, 1998.

[14] F.-L. Lewis and V. L. Syrmos. Optimal Control. Wiley, New York, 1995.

[15] O. Marquet, D. Sipp, and L. Jacquin. Sensitivity analysis and passive control of cylinder flow. J. Fluid Mech., 615:221-252, 2008.

[16] D. S. Park, D. M. Ladd, and E. W. Hendricks. Feedback control of von Kármán vortex shedding behind a circular cylinder at low Reynolds numbers. Phys. Fluids, 6:2390-2405, 1994.

[17] B. Protas. Linear feedback stabilization of laminar vortex shedding based on a point vortex model. Phys. Fluids, 16:4473-4488, 2004.

[18] B. Protas and A. Styczek. Optimal rotary control of the cylinder wake in the laminar regime. Phys. Fluids, 14:2073-2087, 2002.

[19] K. Roussopoulos. Feedback control of vortex shedding at low Reynolds numbers. J. Fluid Mech., 248:267-296, 1993. 